\title{
Psychosis continuum and neurocognition in bipolar disorder
}

\author{
Contínuo de psicose e neurocognição no transtorno bipolar
}

Recent familial and genetic evidence suggest that bipolar disorder and schizophrenia share certain common susceptibility factors. ${ }^{1-3}$ This seems to be especially the case in nonprototypic patients presenting a combination of mood and psychotic symptoms..$^{2-3}$ These findings led to the hypothesis that, unlike non-psychotic bipolar patients, bipolar patients with a history of psychotic features during mood episodes might share certain pathophysiological findings, including brain imaging abnormalities and cognitive impairment.

Although brain imaging and electrophysiology studies have provided some preliminary evidence supporting this psychosis continuum hypothesis; there are very few available studies providing conclusive evidence. ${ }^{3}$ Compared to brain imaging and electrophysiological research, a relatively larger number of studies investigated the effect of a history of psychosis on cognitive abnormalities in patients with bipolar disorder. ${ }^{4}$ In the current issue of Revista Brasileira de Psiquiatria, Brissos et al. ${ }^{5}$ report a key study which examines the cognitive performances of bipolar patients with and without history of psychosis. In this study, the authors compare bipolar patients with healthy controls and patients with schizophrenia. Strengths of the study include recruiting patients in remission and focusing on bipolar I disorder only. The authors did not find a significant effects of psychosis on cognitive abnormalities in bipolar disorder, suggesting that psychosis is not a key clinical determinant of the similarities and differences found in cognitive functioning in schizophrenia and bipolar disorder.

At first glance, this study seems to contradict the bulk findings of previous studies such as a recent meta-analysis that found evidence that a history of psychosis has some effect on cognitive deficits in bipolar disorder. ${ }^{4}$ However, the magnitudes of the cognitive differences in this meta-analysis was quite modest, and effect sizes approached moderate levels for planning and verbal memory abilities only (list learning tasks). In fact, the effect size of the comparison for the Tower of London task $(d=0.55)$ in the study of Brissos et al. ${ }^{5}$ is slightly larger than the most significant difference found in the meta-analysis of Bora et al. ${ }^{4}$ It seems that the current study, like many other individual studies, is underpowered to detect mild to moderate levels of cognitive differences, and that Brissos et al. ${ }^{5}$ also failed to include a list of learning tasks to assess verbal memory. Therefore, it is likely that a history of psychosis might have a modest negative effect on cognitive functioning in bipolar disorder. Psychosis, however, does not operate in isolation of other clinical variables. Psychosis may be a proxy for severity, for how rapidly an episode is recognised and treated, and may be influenced by extraneous factors such as stimulant abuse. It is also possible that modest effects of psychosis on cognitive functioning in bipolar disorder might be related to differences in biomarkers related to illness recurrence ${ }^{6}$ rather than genetic factors, as severe psychotic episodes might be associated with more significant changes in inflammation, neurotrophins and oxidative stress.

The rather modest effects of a history of psychosis on cognitive functioning in bipolar disorder contradict the genetic and familial findings, which indicate a substantial overlap of schizophrenia with psychotic mood disorders, but not with non-psychotic mood disorders. How can we reconcile the contradicting effects of psychosis on cognitive and genetic findings in bipolar disorder? It is unlikely that the sole presence of a history of psychosis will serve as a robust marker of the shared neurobiological risk factors of schizophrenia and bipolar disorder. In fact, this may not be surprising as the familial overlap rate of schizophrenia and bipolar disorder ${ }^{1}$ is much lower than the prevalence of the history of psychosis in bipolar I (approximately $2 / 3$ of the patients had at least one single episode with psychotic features). It can be hypothesized that only a subgroup of psychotic bipolar cases lie in a continuum with schizophrenia and that the content and nature of psychotic symptoms, rather than their mere presence, might be important. Patients presenting with Schneiderian symptoms, paranoid delusions, mood incongruent delusions, and hallucinations are more likely to share neurobiological factors - including cognitive deficits - with schizophrenia, suggesting a spectral relationship. For example, psychotic bipolar cases that would be diagnosed with schizoaffective 
disorder according to research diagnostic criteria (RDC) have a greater probability of showing more pronounced cognitive deficits. In fact, a voxel-based morphometry study suggested that gray matter abnormalities are more severe in psychotic bipolar patients when definition required the presence of persecutory delusions. ${ }^{7}$ Therefore, further studies are needed to examine, in more detail, psychosis-cognition in bipolar disorder and its relationship to shared neurobiological markers with schizophrenia.

Emre Bora ${ }^{1}$, Michael Berk²

${ }^{1}$ Melbourne Neuropsychiatry Centre, Parkville Australia Department of Psychiatry, University of Melbourne, Australia ${ }^{2}$ Deakin University of Melbourne, School of Medicine, Barwon Health, Geelong, Australia. Orygen Youth Health Research Centre, Parkville, Australia. Mental Health Research Institute, Parkville, Australia. Department of Psychiatry, University of Melbourne, Australia

\section{Disclosures}

Emre Bora

Employment: University of Melbourne, Melbourne Neuropsychiatry Centre, Parkville, Australia.

Michael Berk

Employment: Deakin University, Australia.

* Modest

** Significant

*** Significant: Amounts given to the author's institution or to a colleague for research in which the author has participation, not directly to the author.

\section{References}

1. Lichtenstein P, Yip BH, Björk C, Pawitan Y, Cannon TD, Sullivan PF, Hultman CM. Common genetic determinants of schizophrenia and bipolar disorder in Swedish families: a population-based study. Lancet. 2009;373(9659):234-9.

2. Craddock N, Owen MJ. The Kraepelinian dichotomy - going, going... but still not gone. Br J Psychiatry. 2010;196:92-5.

3. Bora E, Yucel M, Fornito A, Berk M, Pantelis C. Major psychoses with mixed psychotic and mood symptoms: are mixed psychoses associated with different neurobiological markers?" Acta Psychiatr Scand. 2008:118(3):172-87.

4. Bora E, Yücel M, Pantelis C. Neurocognitive markers of psychosis in bipolar disorder: a meta-analytic study. J Affect Disord. 2010:127(1-3):1-9.

5. Brissos S, Dias VV, de Souza MGS, Balanzá-Martínez V, Kapczinski F. The impact of past psychotic symptoms on cognitive function in euthymic bipolar patients: a comparison with schizophrenic patients and healthy controls. Rev Bras Psiquiatr. 2011:33(4)353-361.

6. Berk M, Kapczinski F, Andreazza AC, Dean OM, Giorlando F, Maes M, Yücel M, Gama CS, Dodd S, Dean B, Magalhães PV, Amminger P, McGorry P, Malhi GS. Pathways underlying neuroprogression in bipolar disorder: focus on inflammation, oxidative stress and neurotrophic factors. Neurosci Biobehav Rev. 2011:35(3):804-17.

7. Tost H, Ruf M, Schmäl C, Schulze TG, Knorr C, Vollmert C, Bosshenz K, Ende G, Meyer-Lindenberg A, Henn FA, Rietschel M. Prefrontal-temporal gray matter deficits in bipolar disorder patients with persecutory delusions. J Affect Disord. 2010:120(1):54-61. 